\title{
Aerodynamic Characteristics of Isolated Loaded Tires with Different Tread Patterns: Experiment and Simulation
}

\author{
Haichao Zhou* ${ }^{*}$, Zhen Jiang, Guolin Wang and Shupei Zhang
}

\begin{abstract}
The current research of tire aerodynamics mainly focus on the isolated and simplified tread tire. Compared with the real complex pattern tire, the tread pattern structure and deformed profile of a loaded tire has a greatly influence on tire aerodynamic drag. However, the mechanisms of the isolated loaded tires with different tread patterns effects on the aerodynamic drag are subjects worthy of discussion. The purpose of this study is to experimentally and computationally investigate the aerodynamic characteristics of three tires 185/65 R14 with different patterns under loaded. A wind tunnel test model was first established using three-dimensional (3D) printing with a ratio of 1:1, and the pressure coefficients $C_{p}$ of the three tires with different patterns are measured. The paper then conducted computational fluid dynamics (CFD) simulations for analyzing the pressure and flow characteristics. The accuracy of CFD simulation is verified by comparing the simulation results with the test results of pressure coefficients $C_{p}$, and they are of good consistency. While, the general analysis of pressure coefficients $C_{p}$ results of the three tires indicates high-pressure area on the windward surface, and occurrence of low-pressure area on the leeward surface, the pressure coefficients $C_{p}$ of all three tires decreased firstly and then increased along in the air flow direction. The authors finally analyzed the effect of tread patterns on the flow field around the tire and revealed the differences between flow characteristics and aerodynamic drag. The results show that, angle of tire lateral groove has great effect on the flow field characteristics such that; the more the angle of lateral groove agrees with the air flow direction, the less the flow separation and flow vortices, and a minimum observable aerodynamic drag. The research provides a guidance for the design of low aerodynamic drag tires, and helps to illustrate the impact of tire aerodynamics on the car body in the future.
\end{abstract}

Keywords: Tire aerodynamic, Wind tunnel test, Numerical simulation, Flow analysis

\section{Introduction}

Due to global warming, governments and manufacturers have been taking an increasing number of active steps to reduce $\mathrm{CO}_{2}$ emissions. In particular, automobile manufacturers and designers are attempting to reduce $\mathrm{CO}_{2}$ emissions because the road transport accounts for 92 percent of $\mathrm{CO}_{2}$ emissions from all transport services [1]. At high speed, overcoming aerodynamic drag is responsible for more than $50 \%$ of fuel consumption [2]. One effective measure for reducing $\mathrm{CO}_{2}$ emissions is to

\footnotetext{
*Correspondence: hczhou@ujs.edu.cn

School of Automotive and Traffic Engineering, Jiangsu University, Zhenjiang 212013, China
}

decrease the vehicle's aerodynamic drag. It is reported that a $5 \%$ reduction in aerodynamic drag on a typical passenger car would result in a reduction of $1.5 \mathrm{~g} / \mathrm{km}$ of $\mathrm{CO}_{2}$ [3]. Scholars have considered various cases for reducing aerodynamic drag, but most studies focus on modifications of car shape and improvements to airflow around the vehicle body [4]. Airflow can be improved with various efforts, such as optimizing side mirrors, hubcaps, and car bodies [5, 6]. These measures have been implemented effectively and $\mathrm{CO}_{2}$ emissions have declined as a result.

However, because the drag contributions from tires and wheels is about $25 \%$ of a vehicle's overall aerodynamic drag, it cannot be ignored $[7,8]$. When the wheel comes in contact with the airflow, the flow field structure 
undergoes a noticeable change due to tread pattern impact, and three main vortex structures occur-jetting, horseshoe, and top vortices [9]. These vortices would increase the flow separation and aerodynamic drag. To decrease the flow separation and reduce vortices, a previous study [10] presented that, a fully covered wheel blocks the flow from entering the wheel, lessens the flow separation, decreases the vortex size, and reduces drag. Wittmeier et al. [11] suggested that, the aerodynamic drag of a vehicle is reduced by more than $5 \%$ when the openings in the rim are covered using a flat surface cover. However, an enclosed wheel impedes the cooling effect of the brake and thus causes potential hazards to vehicle safety. These potential hazards limit the application domain expansion of the method while the wheel is fully covered. Therefore, extensive experimental studies have been conducted to analyze the influences of rim structures on tire aerodynamics. Vdovin [12] presented the relative importance of tire and rim designs for optimizing aerodynamic characteristics. The effects of rim coverage area, fan spokes, spoke sharpness on the flow field, and drag coefficient of a passenger vehicle were investigated by Bolzon et al. [13], and the results indicate that, rounded spoke edges can reduce the drag coefficient. Brandt et al. [14] developed a numerical model for a vehicle with various rim geometries, and elucidated the effects of certain geometric parameters of rim on the flow field and aerodynamic drag.

A tire is the first part of a vehicle that comes in contact with the outside airflow. Thus, the contour shape and tread pattern of tires have a significant influence on the flow pattern around wheel [15]. Even small differences in the tire geometry can cause significant differences in the aerodynamic drag [8]. It was reported that the measured dimensions of 205/55R16 tires differ from specifications by $10 \mathrm{~mm}$, thus resulting in an increased aerodynamic drag of approximately $2 \%$ with the wider tire [16]. Landström et al. [17] compared the aerodynamic effects of different types of tires for a passenger car using a wind tunnel, and the research showed that the type of tire has a significant effect on the aerodynamic drag, it can make the overall drag of a vehicle vary in magnitude by a factor of $5-10$.

Although the wind tunnel is a powerful device for investigating tire aerodynamics $[15,16,18]$, a huge testing facility is required for wind tunnel testing. Moreover, it is difficult to capture some important flow field characteristics, for example the jetting phenomenon [19]. In the last decade, computational fluid dynamics (CFD) simulations are crucial for studying the flow fields of tires, and CFD simulation results can be confirmed with wind tunnel test results [20]. Axon et al. [21] used CFD simulation to calculate the flow around a wheel and referred to the experiments of Fackrell [18]. Similarly, Diasinos et al. [22] conducted an extensive CFD validation study on a wheel geometry proposed, and the overall pressure coefficient in their study closely matched the coefficient proposed by Fackrell. An unsteady RANS (URANS) simulation was conducted by McManus and Zhang [23], and they obtained a high surface pressure, lift force, and drag force, which were in agreement with those of Fackerell. Wittmeier et al. [24] used CFD to analyze the flow fields around tire with different shoulder geometries. The results showed that the aerodynamic performance of a tire can be improved by smoothing the outward shoulder. The most recent development in the prediction of the flow over an exposed wheel through CFD was published by Uddin et al. [25], who investigated the effects of tire design and operational parameters on aerodynamic performance parameters. Palin et al. [26] used a sliding mesh method to simulate tire rotation and obtained a general understanding of tire airflow characteristics. Hobeika et al. [27] used the moving reference frame approach to analyze the effects of tire pattern features on the drag of a passenger vehicle, and suggested that the rain grooves play a significant role in reducing drag. It is noted that the flow around the tire involves contributions from the tread pattern, ground contact, and rotation [28]. A tire simulation should include tread patterns and the contact patch to reflect aerodynamic characteristics around tire and to obtain the various effects of the different pattern features on aerodynamic characteristics. If the flow field around tire with complex pattern was simulated by CFD, a fine mesh should be used to resolve the lateral grooves. Otherwise, a simple rotating wall boundary condition would result in over predicted drag values $[29,30]$.

It should be noted that, the available related literature on aerodynamics of tire represented the contact patch by a cut tire or a convex platform, or a contact patch without tread patterns $[22,28]$. These simplifications resulted in diverse influences on the flow field around the tire. To study the real effects of the tread patterns on aerodynamic characteristics of tire, tire contact behaviors, the tread pattern deformation and the sidewall bend of three tires 185/65 R14 with different tread patterns, namely; straight groove, axi-symmetrical groove and point-symmetrical groove, were obtained by the finite element analysis, and these features were used to build the three-dimensional (3D) printing model in Section 2. The aerodynamic characteristics of the three tires with different tread patterns was conducted by means of a wind tunnel test and CFD simulation in Sections 3 and 4, respectively. In Section 5, the CFD simulation is validated by the wind tunnel test, and the effects of tread pattern on the flow characteristics of the isolated loaded tires are 
discussed in Section 6. In the last section, the conclusions are drawn.

\section{Experimental Model}

The deformation contour and contact patch of a tire mounted on a vehicle have a considerable influence on the airflow field and separation characteristics around that tire. A study revealed that the sidewall profile and aspect ratio have a significant influence on the aerodynamic characteristics of the tire. To reflect deformation contour and contact patch, a finite element model (FEM) of a passenger car tire 185/65 R14 was established, as shown in Figure 1. Detailed information pertaining to the tire model and simulation analysis was presented by Zhou et al. [31].

A radial stiffness analysis was used to validate tire model. Radial deformations of the tire were simulated under different pressures and load values. The stiffness test equipment displayed in Figure 2a was designed by our team according to the radial stiffness test method that had been illustrated in a previous study [32]. Under a load of $4000 \mathrm{~N}$ and an air inflation pressure of $241.3 \mathrm{kPa}$, the largest deformation section $\mathrm{W}_{\mathrm{d}}$ values were $203.5 \mathrm{~mm}$ and $205.2 \mathrm{~mm}$ for the simulation and the experiment, the difference was within $2 \%$. The comparison of the radial deformation results were presented in Figure $2 \mathrm{~b}$. It can be seen that, the simulation results are in agreement with the measured data with an error of $6.07 \%$. This implies that, the tire model is sufficiently accurate for identifying tire deformation and can be used for subsequent aerodynamic analysis.

To completely retain the details of the tire tread pattern and deformation contour while performing the wind tunnel test, the tire model was reconstructed using threedimensional (3D) printing technology with a scale ratio

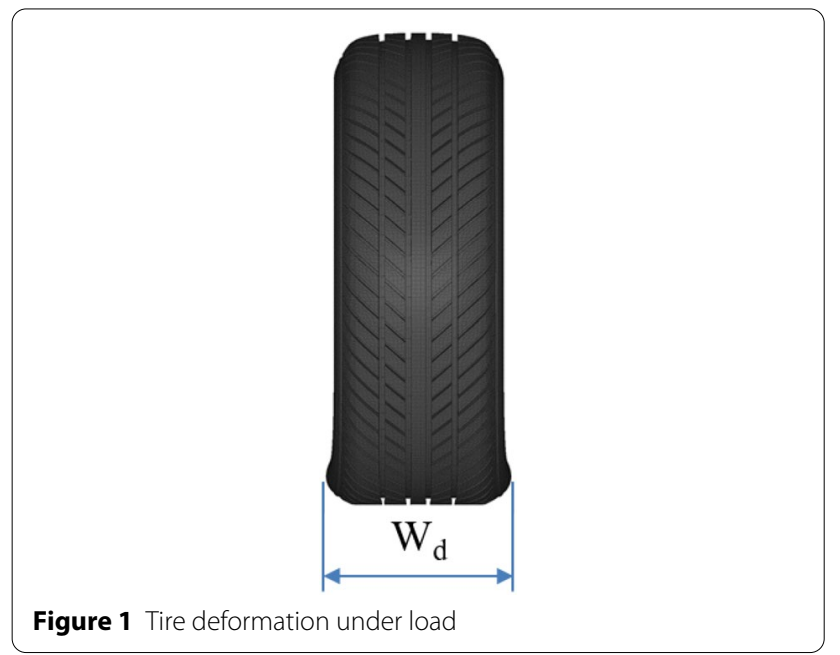

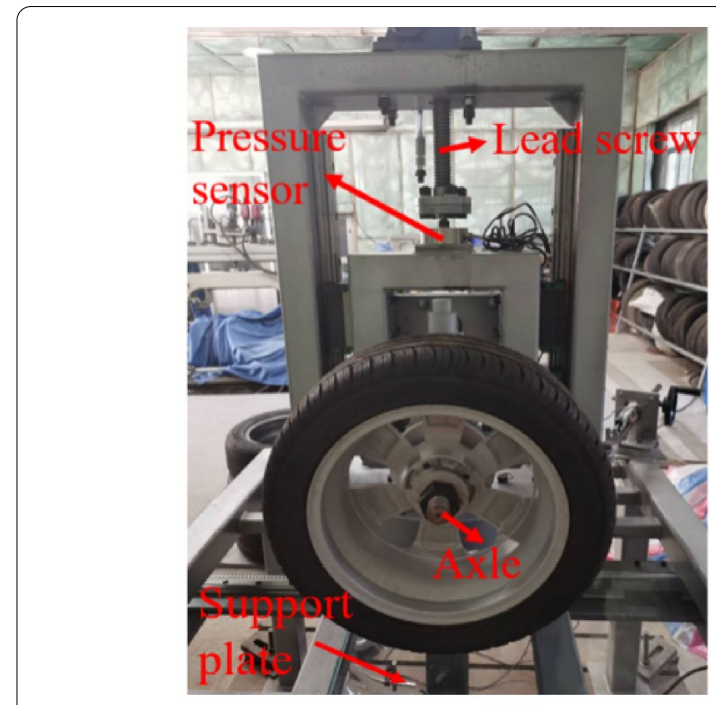

\section{a Stiffness test}

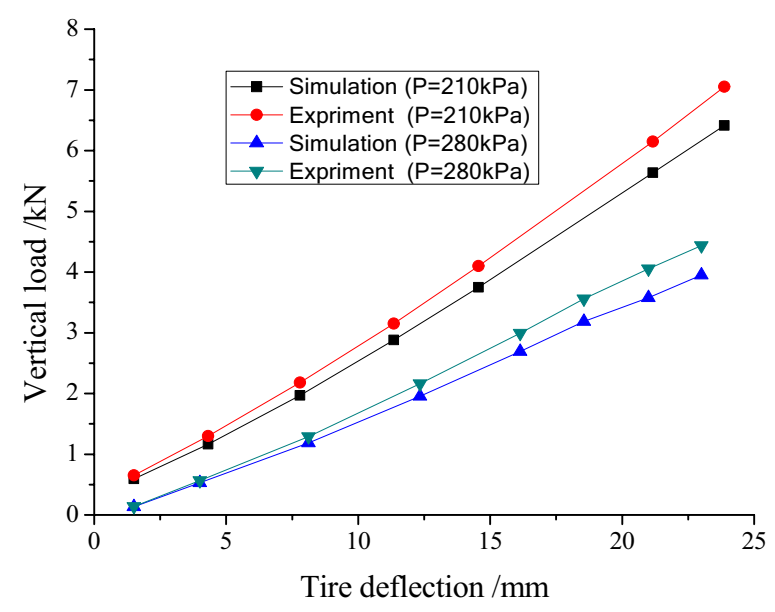

b Stiffness simulation

Figure 2 Tire stiffness test and simulation

of 1:1. SLA resin, which has high strength and rigidity, was used as the printing material.

A tire is mounted on a vehicle within a wheel housing. Approximately only one-third of the tire's height is located outside the wheel housing and is therefore directly exposed to the flow; thus the lowest portion of the tire experiences relatively high air pressure and air stagnation. Although the aerodynamic characteristics of an isolated tire is not the same as those of a tire mounted on a vehicle, the jetting vortices and horseshoe vortices are always presented in the lower half of the tire [28]. What's more, the exposed extent of an actual tire from the ground to the automobile chassis is about $150 \mathrm{~mm}$, 
and the flow field under the vehicle is relatively stable. These results illustrate that, it is significant to analyze the aerodynamic characteristics of the lower half portion of the tire. Thus, a tire model with a height of $150 \mathrm{~mm}$ was used in this study. The basic features of the three tires are shown in Figure 3. The tires displayed in Figure $3 a-c$ are tires with straight grooves, axi-symmetrical grooves and point-symmetrical grooves, respectively. The detailed sizes of the tire model are presented in Figure 3d.

\section{Experimental Setup}

\subsection{Wind Tunnel Setup}

The aerodynamic experiment was conducted at the Wind Tunnel Laboratory of Yangzhou University. The diagram of the wind tunnel effect is displayed in Figure 4 . The wind tunnel is a planar return all-steel structure with high-speed and low-speed testing sections that are arranged in series. The low-speed test section has a length of $7 \mathrm{~m}$, width of $3 \mathrm{~m}$, height of $3 \mathrm{~m}$, wind speed range of $0-25 \mathrm{~m} / \mathrm{s}$, and a turntable with a diameter of 2 $\mathrm{m}$. The high-speed testing section has a length of $3 \mathrm{~m}$, width of $3 \mathrm{~m}$, height of $1.5 \mathrm{~m}$, wind speed range of $0-50$ $\mathrm{m} / \mathrm{s}$, and a turntable of $1.2 \mathrm{~m}$ diameter. The power section is driven by a $185 \mathrm{~kW}$ rating $\mathrm{AC}$ motor with. Air

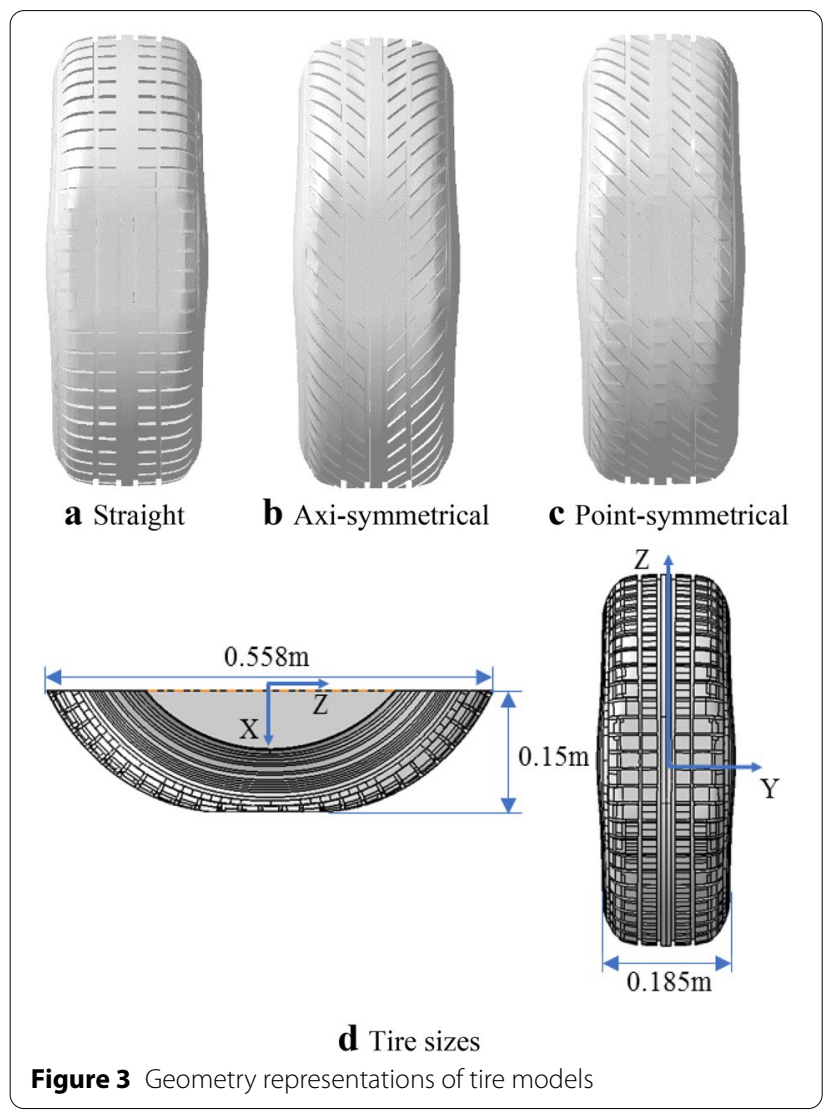

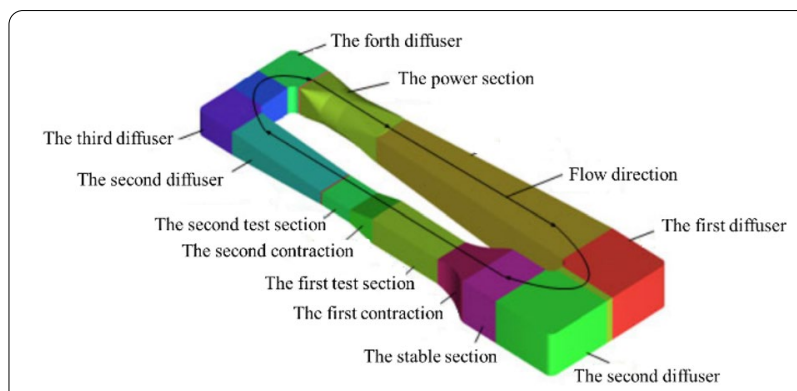

Figure 4 Effect diagram of the wind tunnel at Yangzhou University

temperatures were held constant at $25 \pm 0.5{ }^{\circ} \mathrm{C}$ throughout the testing process.

To fix the tire and reduce the influence of the boundary layer of the bottom wall, the tire was used to form a tire-flat plate model, as shown in Figure 5a. The tire was mounted on the flat plate, and the flat plate was fixed using four supports at the different centers. The center brackets were connected to the flat plate that was fixed with bolts. The front portion of the tire was kept at 20 $\mathrm{mm}$ from the edge of the flat plate, and the center portion of the tire was placed at $0.3 \mathrm{~m}$ from both sides (Figure $5 \mathrm{~b}$ ). The flat plate was placed at a height of $1 \mathrm{~m}$ from the ground. The entire experimental device was installed in the wind tunnel, as shown in Figure 5c. Foam padding was wrapped around the bracket to reduce interference with the flow field.

\subsection{Pressure Measurements}

The surface pressure of the tire was tested using an electronic pressure transducer that has two modules and 128 channels in total (Figure 6a; PSI International, Inc., Virginia, USA). The transducer has a measuring range of $\pm 2500 \mathrm{~Pa}$, maximum sampling frequency of $1200 \mathrm{kHz}$, and measurement accuracy of $\pm 0.05 \%$ full scale (FS). The specific arrangement of piezometric holes is displayed in Figure $6 \mathrm{~b}$. The holes have a diameter of $1 \mathrm{~mm}$, as shown in Figure 6c. One side of the copper pipe of the transducer was mounted on the model, and the other side was connected to an electronic pressure scanning valve by using a plastic pipe.

The sections with the pressure measuring points were almost identical for all three tires used in this study. Taking the straight tire as an example, the tire includes 4 longitudinal sections and 15 lateral sections for each tire model (Figure 7a). The detailed locations of each section are listed in Table 1 and Table 2. The positions of the pressure measuring points in section 1 on the $Y$ axis are shown in Figure 7b. Moreover, the point position distribution for sections 2-4 was the same as that for section 1 . Sections $5,7,9,11,13,15,17$, and 19 were 


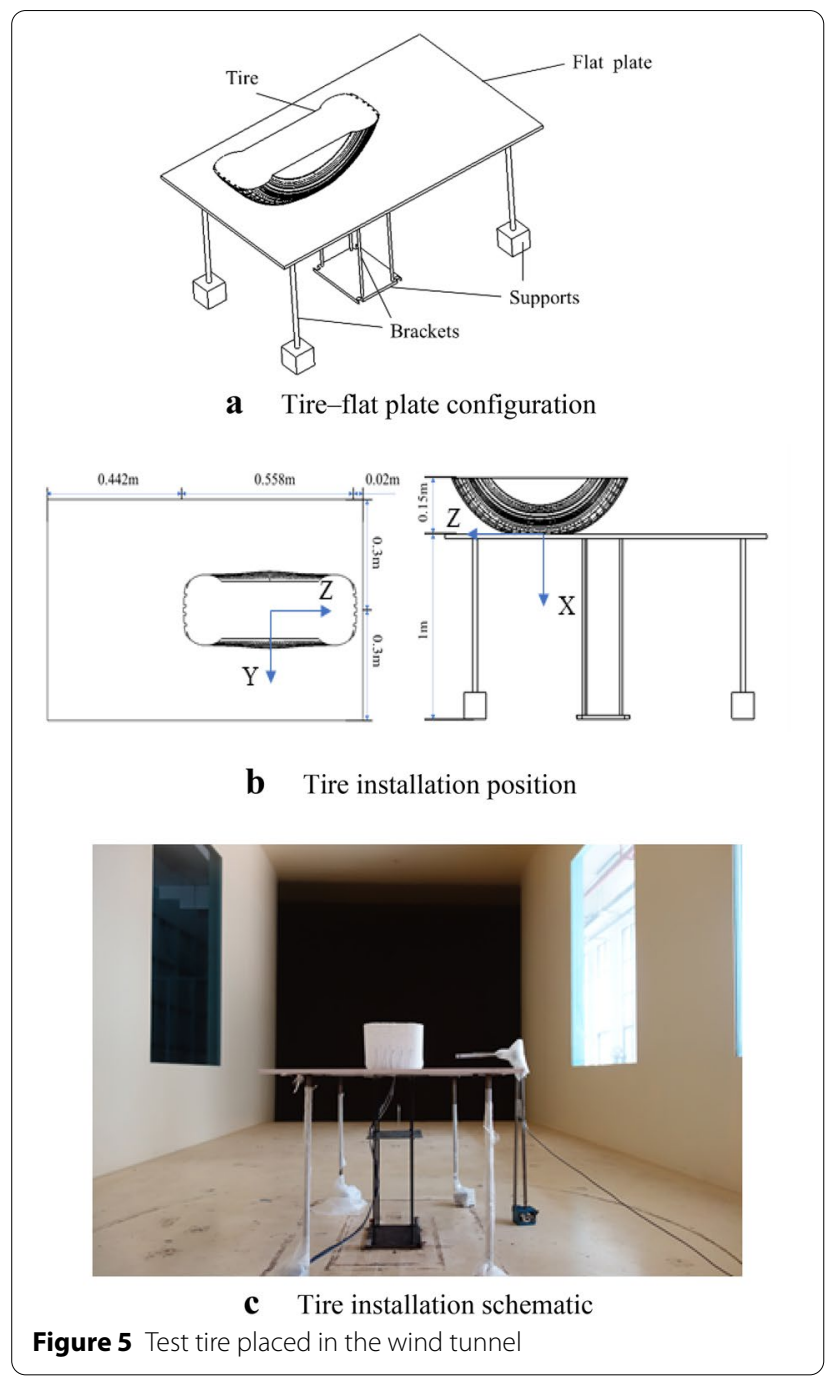

in the lateral tread, and each section of these sections included 7 pressure measuring points. Sections $6,8,10$, $12,14,16$, and 18 were in the lateral groove, and each of these sections included 6 pressure measuring points. The detailed position distribution for lateral sections is shown in Figure 7c-e. Due to the 14 intersection points between the longitudinal sections and the lateral sections in the grooves, the total number of pressure measuring points were 114 .

According to the measured surface pressure of each point, the pressure coefficient $C_{p}$ of each measuring point can be directly calculated. The pressure coefficient $C_{p}$ is a dimensionless parameter with the following specific expression:

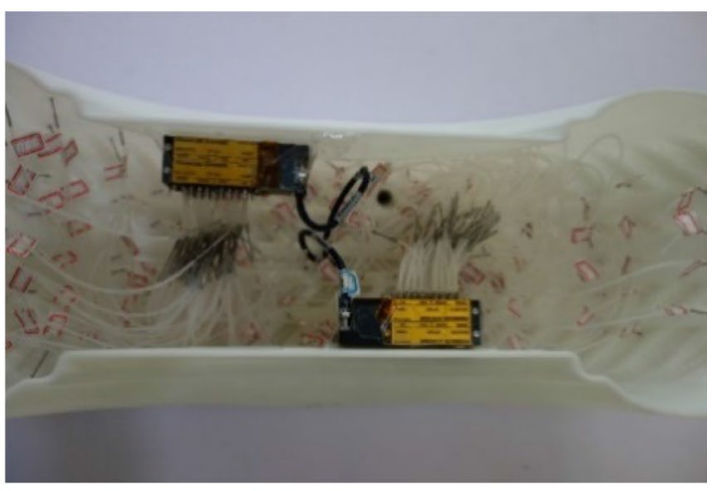

a Pressure transducer

Airflow Tangent

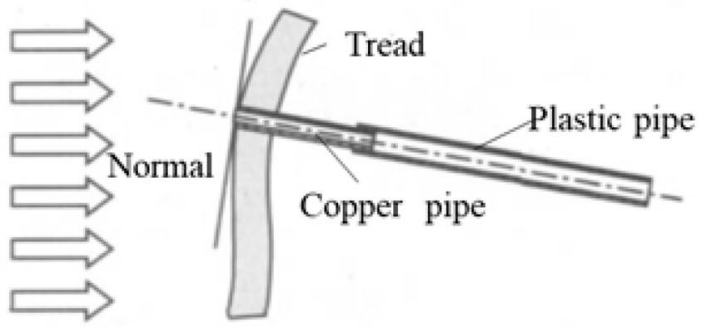

b Layout of the piezometric holes

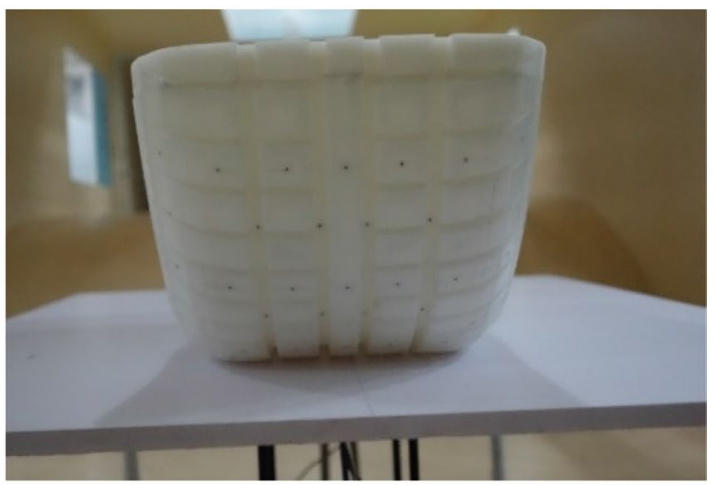

c Pressure measurement points

Figure 6 Tire surface pressure measurements

$$
C_{p}=\frac{p_{i}-p_{r}}{0.5 \rho U_{r}^{2}},
$$

where $P_{i}$ is the measured mean pressure, $P_{r}$ is the reference pressure $101300 \mathrm{~Pa}, U_{r}$ is the wind tunnel airflow speed $15 \mathrm{~m} / \mathrm{s}$, and $\rho$ is air density $1.293 \mathrm{~kg} / \mathrm{m}^{3}$.

\section{Simulation Setup}

\subsection{Computational Domain and Boundary Conditions}

Due to the symmetry of the straight groove and the axisymmetrical grooves, half of the tire geometry model was selected to develop the wind tunnel simulation 


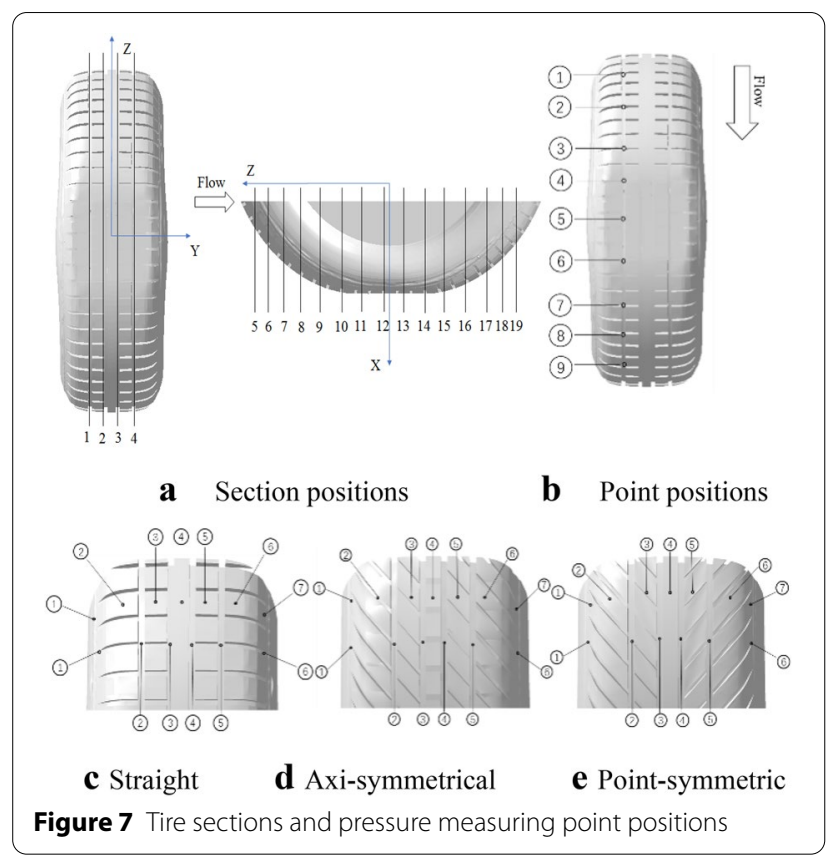

Table 1 Section positions

\begin{tabular}{lllll}
\hline Longitudinal sections & $\mathbf{1}$ & $\mathbf{2}$ & $\mathbf{3}$ & $\mathbf{4}$ \\
\hline$y(\mathrm{~m})$ & 0.011 & 0.0387 & -0.011 & -0.0387 \\
\hline
\end{tabular}

\subsection{Numerical Method}

Two turbulence models were employed for calculating the turbulent stresses, two equations of the shear stress transport (SST) $k-\omega$ model $[33,34]$, and improved delayed detached eddy simulation (IDDES) model [35]. The SST $k-\omega$ model is a two-equation Reynolds average Navier-Stokes (RANS) model that improves the separation prediction in the near-wall region. IDDES is hybrid RANS-LES model that provides a flexible and convenient scale-resolving simulation model for airflow with a high Reynolds number.

All numerical simulations were conducted using the double precision mode of ANSYS Fluent software. Initially, a steady state calculation was conducted using the SST turbulence model. The steady simulation was conducted until the residual errors were reduced below a normalized value of $10^{-4}$, which required approximately 2000 iterations. Then, the steady simulation results were used for initializing the computational domain for the unsteady simulation, and airflow fields were predicted using the IDDES model.

The time step $\Delta t$ was set at $0.0001 \mathrm{~s}$ for the unsteady simulation. These simulations provided the corresponding mean and maximum Courant-Friedrichs-Lewy (CFL) numbers of 0.001 and 2, respectively. The total number of time steps was 10000. A few cells around the tire had CFL numbers larger than 1 . However, the number of cells was so small that this phenomenon was

Table 2 Section positions

\begin{tabular}{|c|c|c|c|c|c|c|c|c|c|c|c|c|c|c|c|}
\hline Lateral sections & 5 & 6 & 7 & 8 & 9 & 10 & 11 & 12 & 13 & 14 & 15 & 16 & 17 & 18 & 19 \\
\hline$z(m)$ & 0.244 & 0.221 & 0.19 & 0.16 & 0.125 & 0.09 & 0.026 & -0.013 & -0.05 & -0.09 & -0.125 & -0.16 & -0.19 & -0.221 & -0.244 \\
\hline
\end{tabular}

model for improving the computational efficiency. However, a complete geometrical model was developed for the point-symmetrical grooves. The computational domain, refinement boxes, and boundary conditions are displayed in Figure 8. The computational domain was $7 \mathrm{~m}$ long, $1.5 \mathrm{~m}$ wide, and $3 \mathrm{~m}$ high. The inlet boundary was $3 \mathrm{~m}$ upstream of the tire, and the outlet boundary was $4 \mathrm{~m}$ downstream of the tire. One mesh refinement box was prepared to ensure that the turbulent flow around the tire was efficiently solved. The detailed setup for the boundary conditions is presented in Table 3.

The computational setting was similar to the static experiment setting. A zero-pressure condition was used at the outlet boundary, and non-slip conditions were used for the tire, table, and wall surface. An air velocity inlet condition of $15 \mathrm{~m} / \mathrm{s}$ was adopted at the inlet boundary. A symmetric boundary condition was used for the symmetric surface.

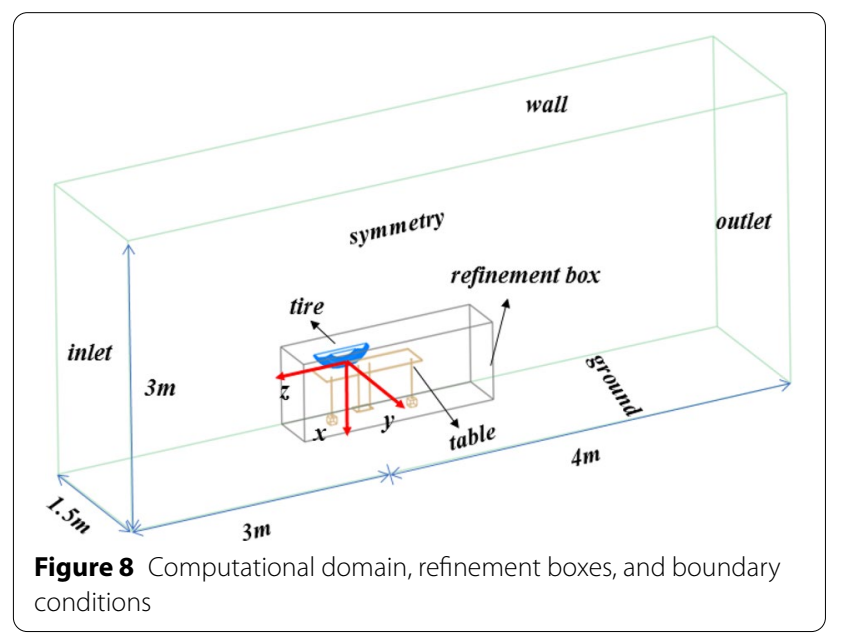


Table 3 Boundary conditions

\begin{tabular}{lll}
\hline Boundary & Boundary condition & Setting values \\
\hline Inlet & Velocity-inlet & $15 \mathrm{~m} / \mathrm{s}$ \\
Outlet & Pressure-outlet & $\begin{array}{c}\text { Standard atmospheric } \\
\text { pressure }\end{array}$ \\
Tire, table, wall & Wall & Non-slip wall \\
Symmetric surface & Symmetry & Standard symmetric surface \\
\hline
\end{tabular}

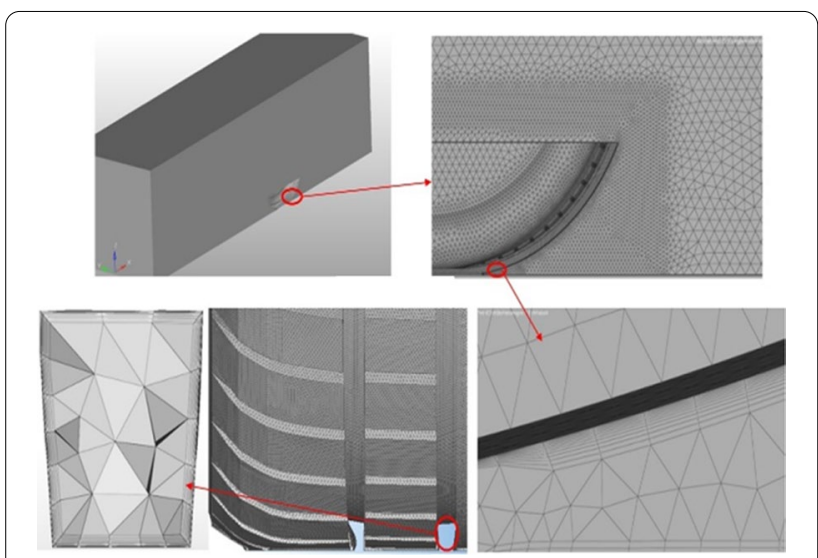

Figure 9 Flow domain and the point-symmetrical tire mesh

considered to have a negligible influence on the flow field.

\subsection{Mesh Description and Verification}

The computational domain comprised meshes formed using tetrahedral elements, and all the elements were generated using the HyperMesh software. Figure 9 presents a general overview of the cell sizes used for each domain. The meshes of the key regions-tire grooves, grounding area, and wake-were refined to ensure calculation accuracy.

Three grids were analyzed: (a) a coarse grid with 4.201 million cells, (b) medium grid with 8.863 million cells, and (c) a fine grid with 14.56 million cells. The distance between the tire surfaces of the first cell to the wall was $0.05 \mathrm{~mm}$, expansion ratio was 1.1 , and total number of boundary layers was 10 . This configuration provided an average non-dimensional wall distance $\mathrm{y}^{+}$of approximately 2 . The base sizes of the coarse, medium, and fine grids were 3,1 , and $0.5 \mathrm{~mm}$, respectively. Moreover, the corresponding maximum sizes on the tire surface were 8,5 , and $3 \mathrm{~mm}$, respectively. Table 4 presents different mesh sizes and number of cells. All meshes in the boundary layers had the same size in the normal direction. The meshes on the tire surface had different sizes in the normal direction. The generated medium mesh of the point-symmetrical tire is displayed in Figure 9.

Mesh sensitivity was investigated using three different meshes-coarse, medium, and fine. The two sections selected for testing mesh sensitivity were longitudinal section 1 and lateral section 9 of the straight tire model. The pressure coefficients $C_{p}$ obtained using the IDDES model with different meshes were compared, as shown in Figure 10. Comparatively, the mesh size had few effects on the windward surface pressure, but affected the pressure on the contact patch and on the leeward surface at a higher amount. The pressure obtained using the medium mesh was consistent with that obtained using the coarse mesh in terms of magnitude and variation, which was in agreement with the experimental results. Differences were noted between pressure coefficients $C_{p}$ obtained using the medium mesh and fine mesh in the contact patch. The $C_{p}$ of the fine mesh obtained was higher than that obtained through experiment. The numerical approaches in the study accurately reproduced the pressure on almost all positions of section 1 except for the contact patch. A certain discrepancy between numerical and experimental results was observed in the contact patch, as shown in Figure 10a. Because the tread groove deformations in the contact patch were complex and irregular, slight differences existed between the tread groove geometry deformations used in the experiment test and CFD simulation. These differences caused the discrepancy. Figure $10 \mathrm{~b}$ shows that the mesh size has almost no influence on the pressure distribution in section 9 . Based on the aforementioned analysis, it was concluded that, the medium mesh provided nearly grid-independent results. Therefore, the medium mesh was used in the remainder of this study.

Table 4 Mesh quality parameters for sensitivity analysis

\begin{tabular}{lllll}
\hline Mesh type & Tire surface cells size $(\mathbf{m m})$ & Thickness of first layer $(\mathbf{m m})$ & Number of boundary layers & Number of total cells \\
\hline Coarse & $3-8$ & 0.05 & 10 & 4.2 million \\
Medium & $1-5$ & 0.05 & 10 & 8.8 million \\
Fine & $0.5-3$ & 0.05 & 10 & 14.5 million \\
\hline
\end{tabular}




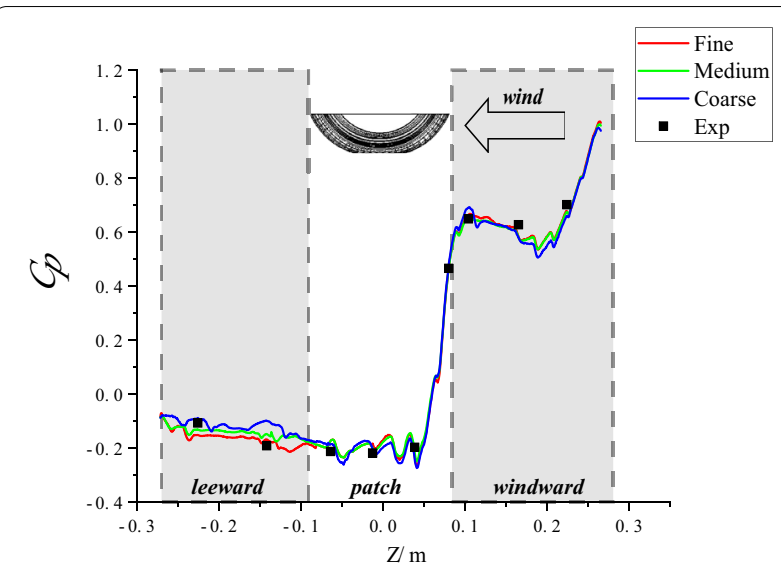

a Section $1(Y=0.011 \mathrm{~m})$

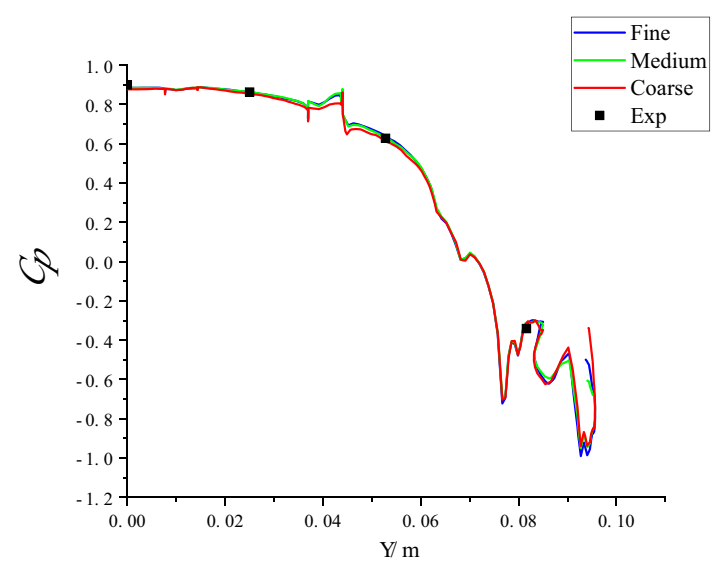

b Section $9(Y=0.0387 \mathrm{~m})$

Figure 10 Time-averaged pressure distribution between three meshes using straight tire model

\section{Simulation Validation}

\subsection{Pressure Comparison of Different Sections}

The time-averaged surface pressure was computed. Figures 11,12 , and 13 display a comparison between the $C_{p}$ values on each longitudinal groove (sections 1 to 4 ) for the three tires obtained using experimentation and numerical simulation. Because the tread grooves of the straight tire and the axi-symmetrical tire were symmetric, only the $C_{p}$ values of one side (sections 1 and 2) were presented.

The simulation results of section 1 and section 2 for the three tires revealed the same trend. That is, the high-pressure area was on the windward surface, and the low-pressure area was on the leeward surface. On the whole, first, the pressure coefficient $C_{p}$ decreased negatively along the $Z$ axis and then increased when it reached the lowest point. This coefficient finally converged on a stable value. Moreover, on the windward surface, a high-pressure was

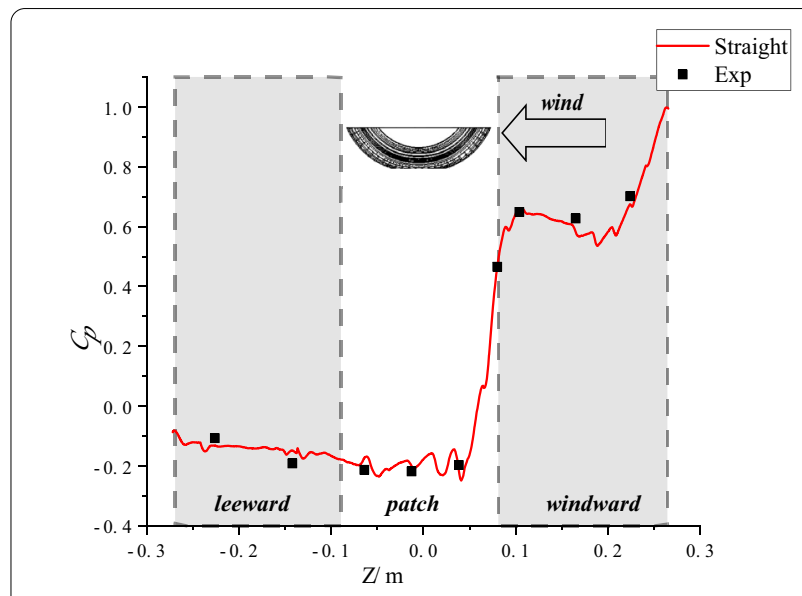

a Section $1(Y=0.011 \mathrm{~m})$

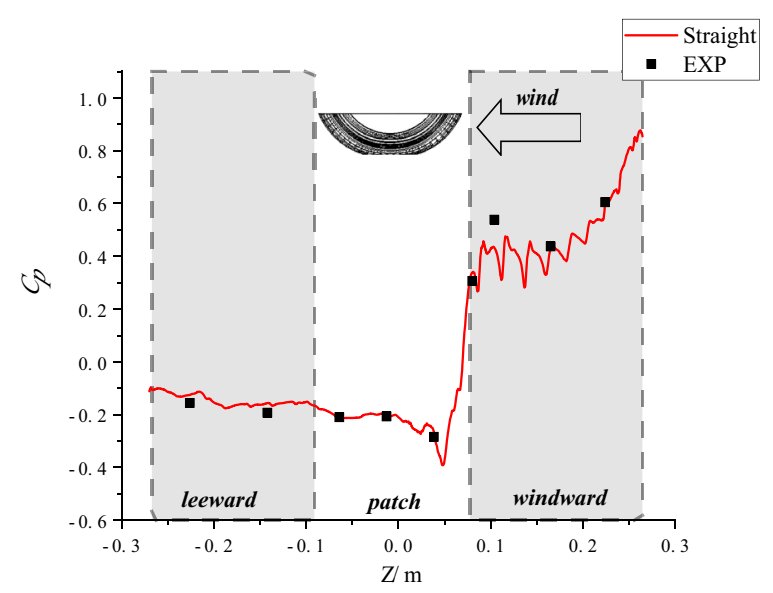

b Section $2(Y=0.0387 \mathrm{~m})$

Figure 11 Comparison of the time-averaged pressure distribution calculated using simulations and experimentation for the straight tire

noted for the section located in the "horn effect" area of the tire-ground contact, where the clearance between the tread and the ground is small, airflow is insufficient, and pressure rises due to the reflection between the ground and the tread. However, for the axi-symmetrical and point-symmetrical tires, the $C_{p}$ values of the longitudinal grooves in the central region continuously decreased on the windward surface. Such a trend does not appear in the straight tire. It is noted that the point-symmetric tread is non-symmetry in the tire width direction. When the air flow inside the point-symmetric tread grooves, the differences of the air flow characters in different positions will lead to the differences of surface pressure.

In general, the experimental test results of the straight tire were in good agreement with the simulation results. 


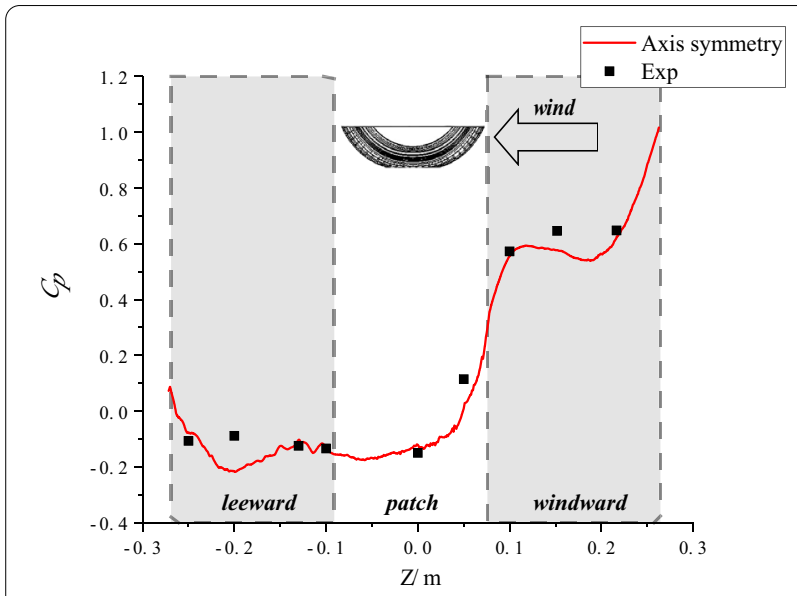

a Section $1(Y=0.011 \mathrm{~m})$

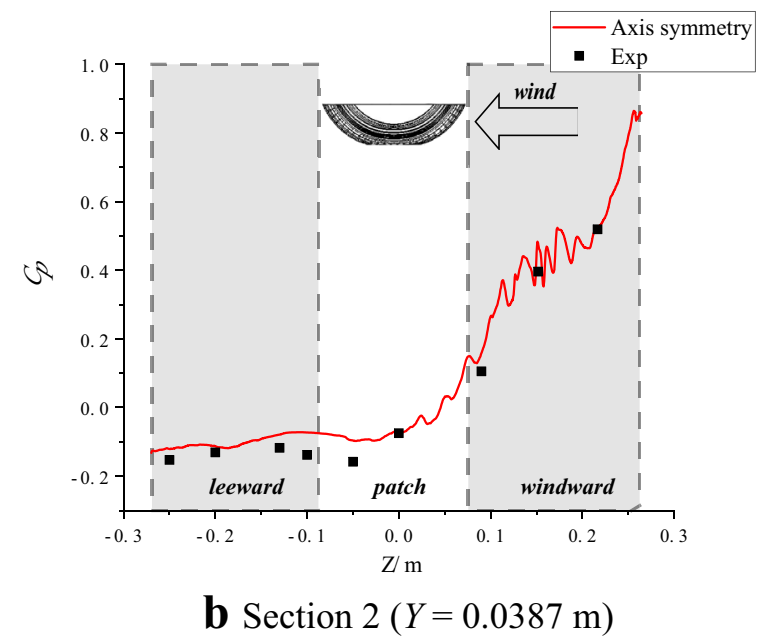

Figure 12 Comparison of the time-averaged pressure distribution calculated using simulations and experimentation for the axi-symmetrical tire

However, the simulation results of the axi-symmetrical and point-symmetrical tires revealed a slight deviation from the experimental results. The deviation mainly occurred at the front and back end of tire contact patch $(Z=-0.1 \mathrm{~m}$ and $Z=0.1 \mathrm{~m})$, as shown in Figures 12 and 13 . The primary reason for the deviation was the geometrical structure simplification applied to the grounding area of the axi-symmetrical tire for ensuring high mesh quality and the convergence of the CFD results. The small number of measuring points near the grounding area caused a difference between the experimental and simulation results. However, on the whole, the pressure coefficient $C p$ distributions of all the three tires were similar and exhibited similar trends. The

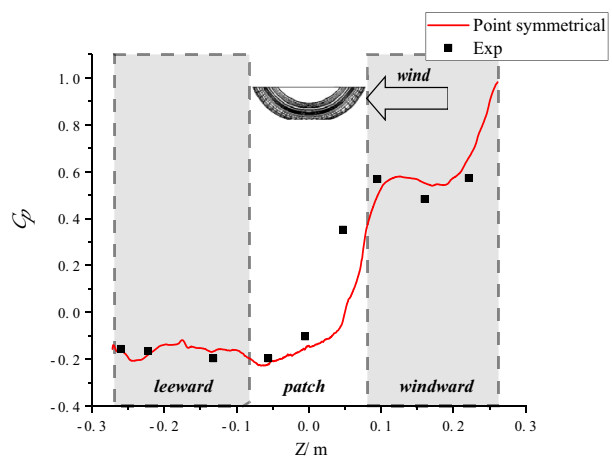

a Section $1(Y=0.011 \mathrm{~m})$

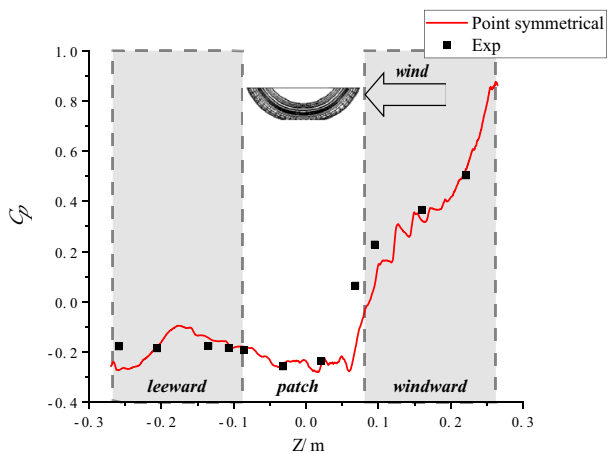

b Section $2(\mathrm{Y}=0.0387 \mathrm{~m})$

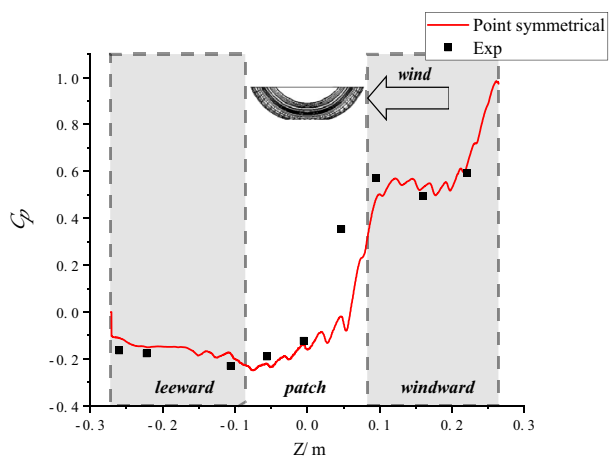

c $\operatorname{Section} 3(Y=-0.011 \mathrm{~m})$

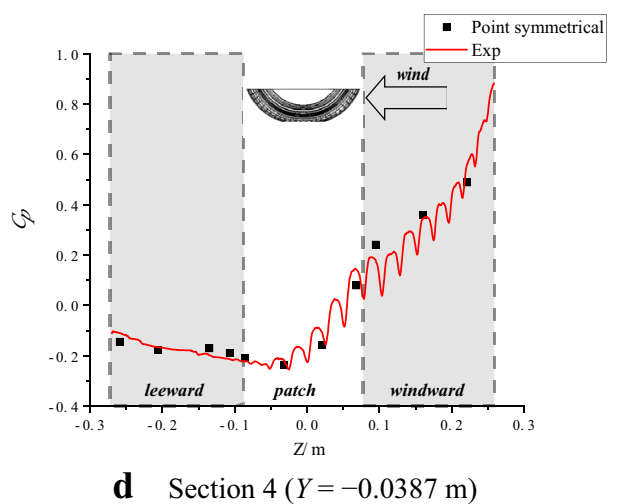

Figure 13 Comparison of the time-averaged pressure distribution calculated using simulations and experimentation for the point-symmetrical tire 
simulation results were in good agreement with results from experiment.

\subsection{Comparison of Streamline}

During the wind tunnel test, a thin rope was attached at the longitudinal section ' 1 ' of the point-symmetrical tire, $75 \mathrm{~mm}$ away from the flat plate for obtaining detailed flow field information. The motion state of the thin rope observed when the airflow in the wind tunnel is stable is displayed in Figure 14a. Figure 14b presents the CFD simulation result. The tail streamline obtained using CFD was oblique and smooth, which is in good agreement with the experimental results.

Knowles [36] conducted surface pressure measurements of planes located at $0.54,0.60,0.69$, and 0.88 of wheel diameter in the downstream of the wheel center to determine the wake structure. This experiment was only conducted for a slick tire without investigating the effect of tread pattern on the airflow structure. The position of the plane located at a diameter of plane $\mathrm{A}$ in this paper is shown in Figure 15a. Figure 15b-e reveal the airflow results obtained using the CFD simulation and experimental test presented by Knowles on a plane located at a diameter of plane A. The CFD simulation reproduces the main characteristics of the flow field, which captures the main vortices. The shape of the flow structure is also consistent with the flow structure results of previous experimental studies conducted by Knowles [36], and Saddington [37], as presented in Figure 15e, f. The downward deflection of the jet stream is clearly observed to form vortices that are similar to those observed in the experimental results. For the difference in the flow field in the upper half, this study mainly analyzed the flow field in the tire grounding area and did not focus on the

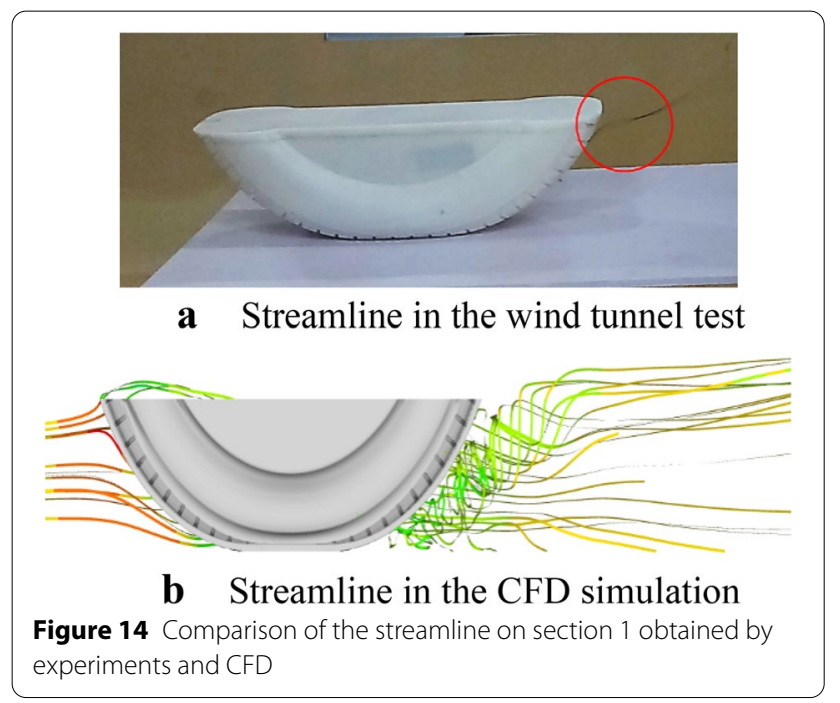

flow field in the upper half of the tire grounding area. Moreover, the sizes of the vortices predicted using CFD simulation were slightly different from the sizes obtained through experimentation. A reason for this difference is that, all of the aforementioned studies have been conducted using non-deformable tire. Another reason that should not be overlooked is, the tires used in this study have complex tread patterns, which can be seen from the Figure 3, and these differences would result in flow field change consistent with the conclusions form Hobeika that tread grooves have a significant effect on the flow field around tires [38].

Comparison of the pressure distributions calculated using simulations and experimentation proves that the test results of all tires approximately match the simulation results. The flow field results are consistent with the findings of a study. The deduction from the compared results suggest that CFD simulation can accurately predict the main flow field characteristics. Hence, the next section analyzes the effect of tread patterns on flow fields by using the above computational details.

In order to ensure consistency in the analysis of aerodynamic drag between the partial tire models presented in this paper and the entire tire models, the drag coefficient $C_{\mathrm{d}}$ is computed using the CFD simulation mentioned above. The comparison of $C_{\mathrm{d}}$ shown in Figure 16 indicates that, the drag coefficient $C_{\mathrm{d}}$ of the partial model agrees well with that of the entire model. It proves that the partial model has a certain research value in the analysis of tire aerodynamics.

\section{Flow Result Analysis}

Traditionally, behaviors of the vortex, which can be equivalently expressed by motions of rotation, are often regarded as turbulence structures. In addition, the quantitative magnitude of the rotation can be estimated by the Q-criterion. Proposed by Hunt in 1988 [39], the Q criterion is a powerful method for examining variations in the rotation structures at a wide range. The $\mathrm{Q}$ criterion is one of the best methods used for identifying $3 \mathrm{D}$ vortices. In this study, the $\mathrm{Q}$ criterion was used to describe a turbulent flow field. Figure 17 presents the iso-surface of the $Q=3000$ of the straight, axi-symmetrical, and pointsymmetrical tires, respectively. The formation of the isosurface of the $\mathrm{Q}$ validates the length in the $Z$ direction to be more suitable for developing complete flow structures.

In the flow direction, air meets tire and turn flow directions close to the sidewall region. As well, air flow past tread grooves. As a result, a large number of lumps of the iso-surface of the $\mathrm{Q}$ are observed around the rear contact patch. It can be seen from Figure 17 that, the distribution of lumps of the iso-surface of the $\mathrm{Q}$ have significant differences due to the different tread pattern. 


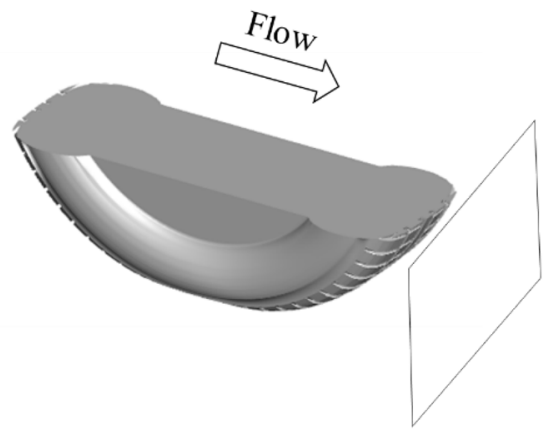

a Position of the plane with a diameter of plane A

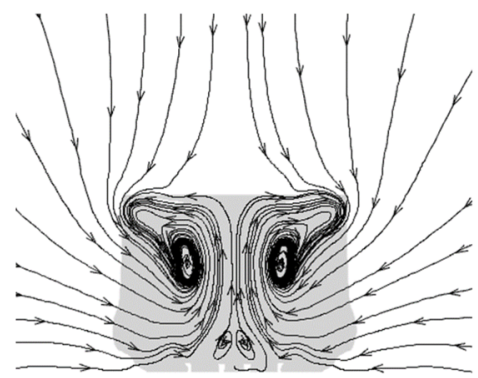

c Axi-symmetrical flow field

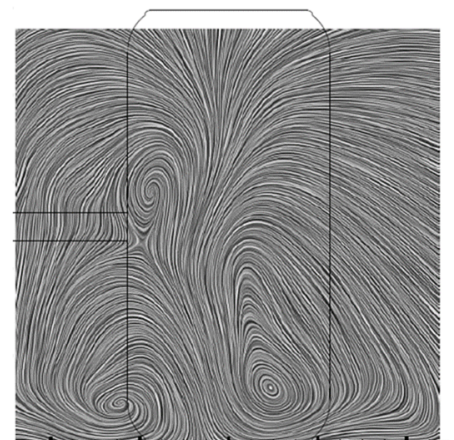

e Experiment test results by Knowles [36]

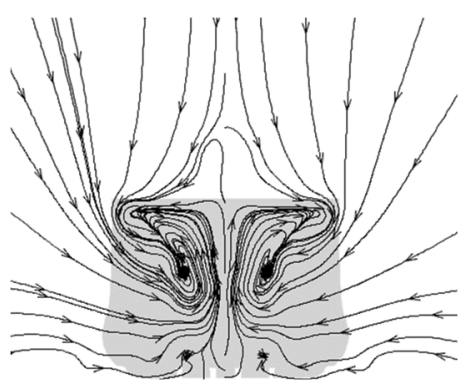

b Straight groove flow field

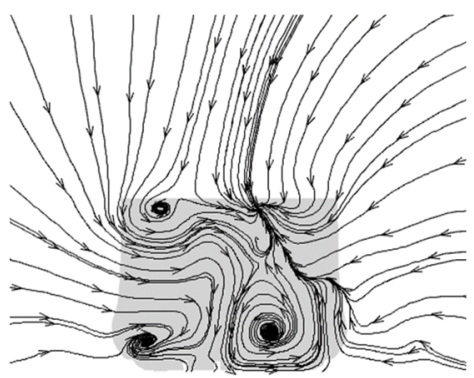

d Point-symmetrical flow field

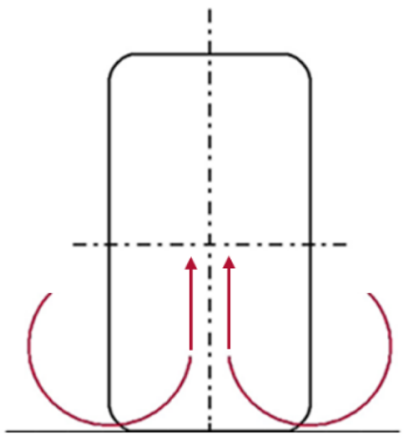

f Experimental results by Saddington [37]

Figure 15 Comparisons for flow filed

The axi-symmetrical tread is apparently denser than that of the large magnitude of lumps of the iso-surface of the Q shown in the three tread patterns; on the contrary, the point-symmetrical tread has the sparsest of the magnitude of lumps of the iso-surface of the $\mathrm{Q}$. The reason for this difference is that, the point-symmetrical grooves is interconnected in the air flow direction, and this makes air easier flow past it; the axi-symmetrical grooves is in the opposite direction of the incoming air flow, and this is not beneficial to air flow. Vortices develop due to the shear force when a flow passes the tires. When the airflow reaches the tire, the flow field structure performs a noticeable change and two main vortex structures around the tire occurs, i.e., jetting vortices and horseshoe vortices. Various jetting vortices were observed for all tires with all tread patterns, but the horseshoe vortices was the predominant vortex structure behind the tire contact patch, and the horseshoe vortices highly influences the global flow around the tire.

Because of the different symmetrical forms of tread patterns, straight and axi-symmetrical tires present symmetric vortices distributions. Moreover, the pointsymmetrical tire presents an asymmetric vortices distribution. The weak structure of the straight tire has a wide distribution range for which the flow at the end of the wake diffuses outward. The weak structure of the 


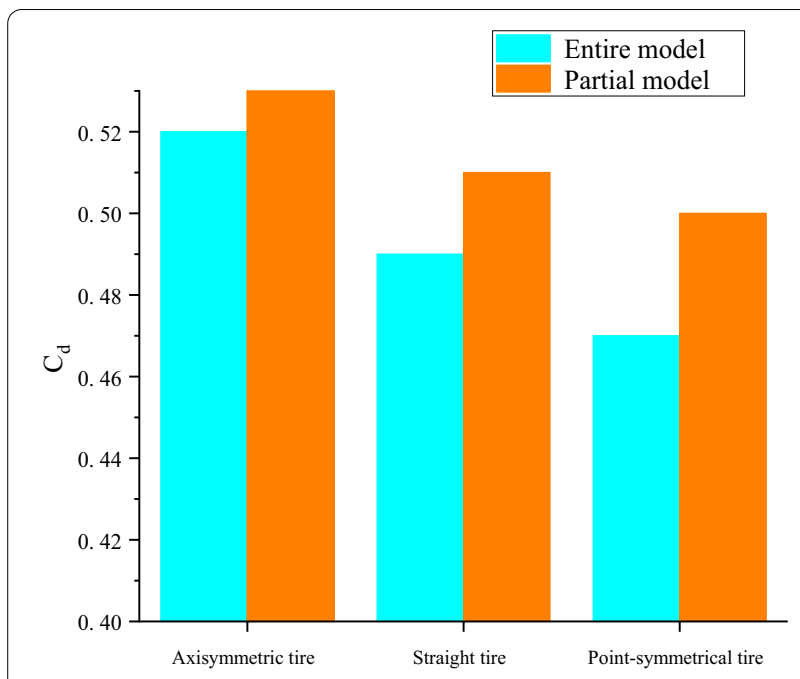

Figure 16 Comparison of resistance coefficient $C_{d}$ for three tires

axi-symmetrical tire distribution range is smaller than that of the straight tire distribution and is accumulated near the centerline. But the vortex distribution of the axisymmetrical tire is denser than that of the straight tire. The weak structure of the point-symmetrical tire distribution range is much smaller than that of the other two tires.

The vortex is caused by the interference and separation of flow on the leeward surface. The generation of vortex is accompanied by the loss of mechanical energy. Controlling vortex shedding and wake vortices is beneficial to flow improvement and drag reduction. Combined analysis of the aerodynamic drag of the three tires reveal that the axisymmetric tread grooves to be unparalleled to the air flow, and this increases the flow separation and strengthen the generation of vortex, vortex breakdown, and with the shortest vortex line, while the drag is maximum. Combined analysis of the aerodynamic drag of three tires, the axi-symmetrical grooves is unparallel to air flow, and it increases flow separation. Oppositely, the point-symmetrical tread grooves are parallel to air flow direction, and it reduces flow separation, and the vortex line is longer, and the flow attachment over tire surface causes drag reduction.

The flow on plane A was observed; plane A was located $2 \mathrm{~mm}$ above the ground. Figure $18 \mathrm{a}-\mathrm{c}$ display the pressure and streamlines distributions on plane A for the straight, point-symmetrical, and axi-symmetrical tires obtained using CFD simulations, respectively. The pressure distribution trends of the three tires were almost similar: the high-pressure area was at the front of grounding area and the low-pressure area was at the side in the downstream region.

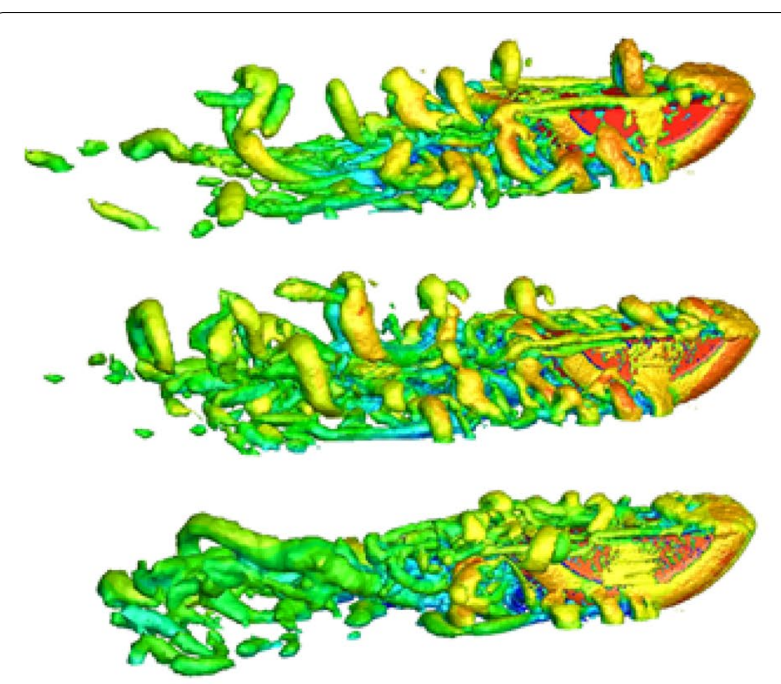

a Main view

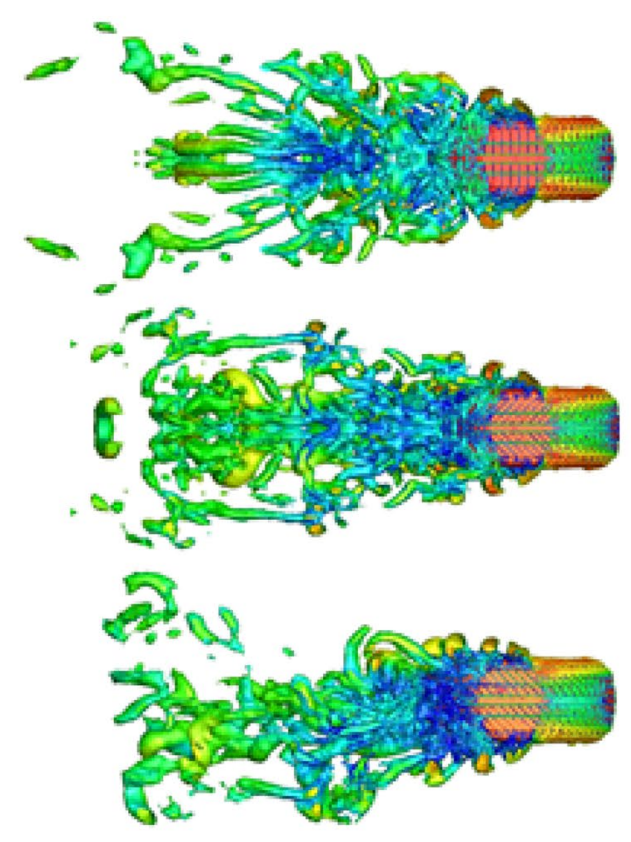

b Upward view

Figure 17 Time-averaged $Q$-criterion iso-surfaces $(Q=3000)$ colored according to the velocity

By observing the streamline distribution presented in Figure 18, it can be seen that the three tires with different tread patterns produce a vortex on both sides of the shoulder. However, the diameter and position of the vortex core were different. Many small vortices were scattered in the straight and axi-symmetrical tires. However, few vortices were scattered in the point-symmetrical tires. The discrepancies are mainly due to the flow 


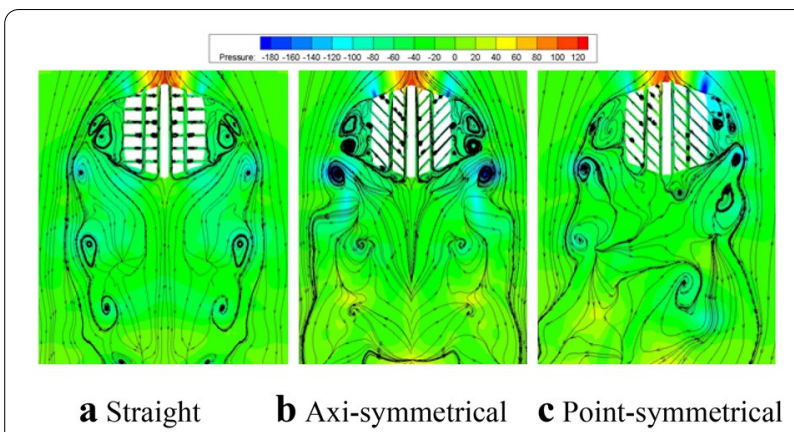

Figure 18 Pressure and streamline distributions on plane A for three tire models

variations because of the lateral groove angle. The lateral groove angle of the straight tire was orthogonal to the airflow direction. Thus, the flow directly impacts the groove wall, and the vortices are generated by the shear force of the wall (Figure 18a). The flow field structure of the axisymmetrical tire is similar, but it has obvious vortices at the outlet of the lateral grooves mainly because the direction of flow is almost opposite to that of the inflow (Figure18b). Fewer vortices are present in the right lateral grooves of the point-symmetrical tire when the right lateral grooves angle is near to the inflow direction (Figure 18c). Moreover, the straight tire produces wake vortices along the centerline. These features are obvious and are present even far away from the centerline. Conversely, the wake of the axi-symmetrical tire is larger than that of the straight tire and closer to the exit of the longitudinal groove. Compared with the other two tires, the wake vortices of the point-symmetrical tire are smaller and unevenly scattered on both sides. The vortex is caused by flow separation. The denser the vortex distribution, the greater the degree of boundary layer separation, and the higher the pressure drag. Based on the pressure contours and Bernoulli's equation for the three tire, although a high pressure area is associated with the windward, the pressure of the axisymmetric tire in the leeward is minimum, and the pressure of the point-symmetric tire in the leeward is maximum. From a comprehensive analysis of the pressure contours with streamlines, the drag of the axisymmetric tire is maximum, whereas that of the pointsymmetric tire is minimum.

Figure $19 \mathrm{a}-\mathrm{c}$ display the time-averaged vorticity distributions on plane A for the straight, point-symmetrical, and axi-symmetrical tires obtained by CFD simulations, respectively. Figure 19 reveals that, there are many vortices in the groove, shoulder, and tail. This indicates that, the airflow in the grounding area is disordered and further indicates the complexity of the flow in the grounding area.

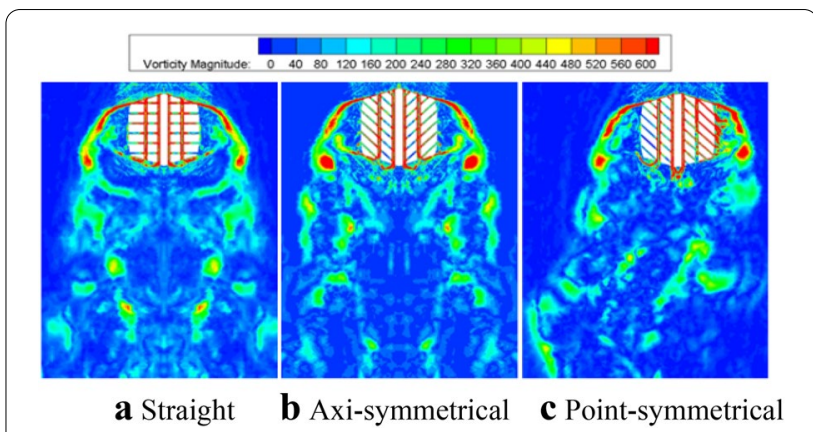

Figure 19 Time-averaged vorticity distributions on plane A for three tire models

Further analysis presents the movement of air in the grooves of the grounding areas of the three tires. Figure 19 reveals that, the movement trends of the airflow in the longitudinal grooves of the grounding areas of the three tires are relatively consistent. However, the movement patterns of the fluid particles in the lateral grooves are relatively obvious. When the fluid flows along the lateral groove, the flow direction is at an angle with the formation of the lateral grooves. Therefore, the flow in the lateral groove constantly impacts the wall. This worsens the flow instability and then leads to an increase in the dynamic pressure in the grounding area, which is not conducive to the flow movement. The evidence proves that, the closer the arrangement of lateral grooves in the grounding area is to the moving direction of fluid particles, the smaller the flow impact on the wall of lateral grooves is, and the smaller the pressure and vortices, the more stable and smooth the flow movement. On the contrary, a larger fluid impact leads to serious perturbation, and a larger area of vortex distribution on the leeward surface. The large range of vortices means that, there is a large amount of wasted energy due to flow separation, resulting in a corresponding drag increase.

The velocity distribution around the tire at different lateral sections is shown in Figure 20. On the windward surface for section 8 of the three tires, there are few differences in velocity contour lines. For the section 12 of the straight and axi-symmetrical tire, although the boundary of velocity contour lines is similar, the velocity gradient of the straight tire is obviously lower compared to the axi-symmetrical tire, indicating that, the fluid shear stress of the straight tire is smaller relative to the axi-symmetrical tire. Because the point-symmetrical tire is asymmetric grooves, the shape of the velocity contour lines of both sides is different. The flow area of low velocity air of the point-symmetrical tire is smaller than that of the straight tire. An indication that, the flow stability of 


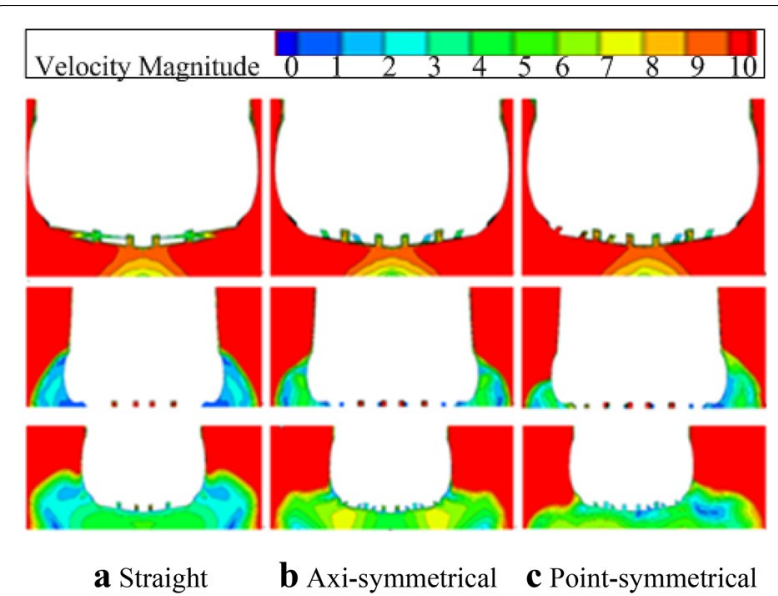

Figure $\mathbf{2 0}$ Velocity distribution around the tires in different lateral sections (Top: section 8; Middle: section 12; Bottom: section 16)

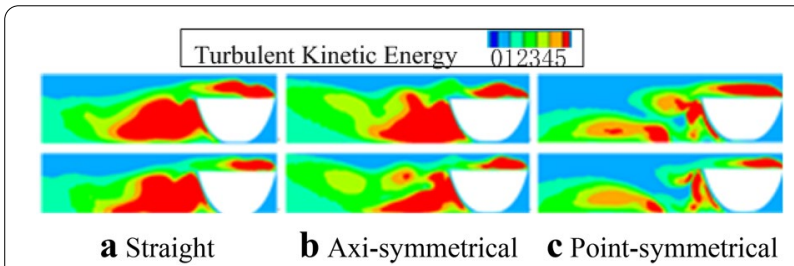

Figure 21 Turbulent kinetic energy around tires at different longitudinal sections (Top: section 1; bottom: section 2)

point-symmetrical tire is better, and the flow vorticity is less (refer to Figure19).

On the leeward surface of the three tires, there are considerable differences observed in the velocity distribution shown in section 16 . The velocity of the axi-symmetrical tire is greater than that of the other two tires. Based on Bernouli's equation, the pressure of the axi-symmetrical tire on the leeward surface is smaller than the pressure recorded for the other two tires (see Figure 18). The region of low velocity of the straight tire is higher than that of the point-symmetrical tire, and more flow vortex was generated on the leeward surface, as shown in Figure 17 . Also the energy dissipation of wake vortices might be enlarged.

It is well known that, the turbulent kinetic energy (TKE) directly represents the strength of the turbulent in the flow, and we compared the TKE in order to investigate the unsteady flow characteristics of flow over the longitudinal sections. Figure 21 compares the TKE of the flow over longitudinal section 1 and section 2. At the same longitudinal section, the turbulent strength of axisymmetrical tire was the largest, and the point-symmetrical tire was the least. The contour of the TKE revealed that, the turbulent strength diminished gradually with distance on the leeward surface. However, the high TKE from the highly unsteady flow in the axi-symmetrical tire is due to lateral grooves impeding fluid flow. The effects of guides direction of point-symmetrical lateral grooves on air flow can reduce turbulent fluctuations in the longitudinal grooves, and its benefit is to reduce the generation of vortex shedding.

\section{Conclusions}

Wind tunnel experiments and numerical simulations were conducted in this study to investigate the aerodynamic characteristics of three tires with different patterns-straight, axi-symmetrical, and point-symmetrical patterns. The pressure coefficients of the three tires were measured in the wind tunnel experiments. In this study, CFD simulations were conducted using the steady SST $k-\omega$ model, followed by the unsteady DES approach.

By comparing the pressure distributions calculated using simulations and experiments from sections 1-4, the simulation results of all three tires were found to agree well with the wind tunnel experiment results. Generally the results of the three tires indicate high-pressure area on the windward surface, and the leeward surface is characterized by low-pressure area. The pressure coefficients $C_{p}$ of all three tires decreased firstly and then increased along the air flow direction. The flow field results presented the main flow characteristics, which are also consistent with previous findings. This reveals that the results of flow field calculation can be used in a follow-up study. The effects of tread pattern on the flow structure were demonstrated according to the numerical simulation results. The flow fields of the three different types of patterned tires are quite different. The closer the arrangement of lateral grooves in the grounding area is to the moving direction of fluid particles, the smaller the impact of fluid is on the wall of lateral grooves, the smaller the vortices are, and the more stable and smooth the flow of fluid is.

This study thus indicates that, the tire lateral groove angle has an effect on the flow field pressure, streamline, and vortex generated. The results demonstrates that tire's lateral groove angle has great effect on the flow field characteristics. The more the angle of lateral groove angle agrees with the air flow direction, the lesser the surface pressure and aerodynamic drag. The numerical and experimental conclusions can offer guidance for designing low aerodynamic drag tires. However, some limitations require attention. Although the influence of the tread pattern on flow field were investigated, the flow field difference between a half tire and real wheelhouse is worth analyzing. Moreover, difference between a rotating and stationary tire was not studied here. These limitations warrant further investigation in the near future. 


\section{Acknowledgements}

The authors sincerely thanks to Professor Yang Hua of Yangzhou University for his critical discussion for the preparation of the wind tunnel test.

\section{Authors' contributions}

$\mathrm{HZ}$ and $\mathrm{GW}$ were in charge of the whole trial, $\mathrm{HZ}$ and $\mathrm{ZJ}$ wrote the manuscript and were in charge of the whole analysis; SZ revised the final manuscript. All authors' read and approved the final manuscript.

\section{Authors' Information}

Haichao Zhou, born in 1984, is currently an associate professor at Jiangsu University, China. He received his Ph.D. Degree from Jiangsu University, China, in 2013. His research interests include tire aerodynamic, tire simulation technology and tire structure optimization.

Zhen Jiang, born in 1994, is currently a master candidate at School of Automotive and Traffic Engineering, Jiangsu University, China.

Guolin Wang, born in 1965, is currently a professor and a Supervisor of Ph.D. candidates at School of Automotive and Traffic Engineering, Jiangsu University, China. His main research areas include automobile engineering, vehicle-terrain mechanics and tire structure design technology.

Shupei Zhang, born in 1981, is currently an associate professor at Jiangsu University, China. He obtained his Doctorate Degree from Jilin University, China, in 2011 . His research interests include automobile systems measuring techniques and intelligent drive techniques.

\section{Funding}

Supported by National Natural Science Foundation of China (Grant Nos. 52072156, 51675240), Jiangsu Provincial Youth Fund of China (Grant No. KB20160528), Jiangsu Provincial Six Talents Summit Program of China (Grant No. JXQC-011), and Jiangsu University Senior Talents Startup Fund (Grant No. 1291120046).

\section{Competing Interests}

The authors declare no competing financial interests.

Received: 24 December 2019 Revised: 12 November 2020 Accepted: 9 December 2020

Published online: 05 January 2021

\section{References}

[1] R M González, G A Marrero, J Rodríguez-López, et al. Analyzing $\mathrm{CO}_{2}$ emissions from passenger cars in Europe: A dynamic panel data approach. Energy Policy, 2019, 129: 1271-1281.

[2] G Sivaral, K M Parammasivam, G Suganya. Reduction of aerodynamic drag force for reducing fuel consumption in road vehicle using basebleed. Journal of Applied Fluid Mechanics, 2018, 11(6): 1489-1495.

[3] THobeika, S Sebben. Tyre pattern features and their effects on passenger vehicle drag. SAE International Journal of Passenger Cars - Mechanical Systems, 2018, 11(5): 1-12.

[4] B Cihan. Numerical drag reduction of a ground vehicle by NACA2415 airfoil structured vortex generator and spoiler. International Journal of Automotive Technology, 2019, 20(5): 943-948.

[5] X Chen, M Li. Delayed detached eddy simulation of subcritical flow past generic side mirror. Journal of Shanghai Jiaotong University (Science), 2019, 24(1): 107-112.

[6] A P Mears, R G Dominy. Racing car wheel aerodynamics - Comparisons between experimental and CFD derived flow-field data. SAE Technical Papers, 2004-01-3555.

[7] J Reiß, L Haag, T Indinger. CFD investigation on fully detailed and deformed car tires. International Journal of Automotive Engineering, 2019, 10(4): 324-331.

[8] K Hirose, H Kawamata, M Oshima. Aerodynamic sensitivity analysis of wheel shape factors. SAE Technical Paper, 2019, 2019-01-0667.

[9] A Huminic, G Huminic. Aerodynamic study of a generic car model with wheels and underbody diffuser. International Journal of Automotive Technology, 2017, 18(3): 397-404.
[10] M E Gleason, A Guzman, B Duncan, et al. Comparison of computational simulation of automotive spinning wheel flow field with full width moving belt wind tunnel results. SAE International Journal of Passenger Cars - Mechanical Systems, 2015, 8(1): 275-293.

[11] F Wittmeier, T Kuthada, N Widdecke, et al. Model scale based process for the development of aerodynamic tire characteristics. SAE Technical Papers, 2014-01-0585.

[12] A Vdovin. Investigation of aerodynamic resistance of rotating wheels on passenger cars. Chalmars University of Technology, Gothenburg, Sweden, 2013.

[13] M D P Bolzon, S Sebben, A Broniewicz. Effects of wheel configuration on the flow field and the drag coefficient of a passenger vehicle. International Journal of Automotive Technology, 2019, 20(4): 763-777.

[14] A Brandt, H Berg, M Bolzon, et al. The effects of wheel design on the aerodynamic drag of passenger vehicles. SAE Technical Papers, 2019-01-0662.

[15] L Haag, T Blacha, T Indinger. Experimental investigation on the aerodynamics of isolated rotating wheels and evaluation of wheel rotation models using unsteady CFD. International Journal of Automotive Engineering, 2017, 8(1): 7-14

[16] C Landström, TWalker, L Löfdahl. Effects of ground simulation on the aerodynamic coefficients of a production car in yaw conditions. SAE Technical Papers, 2010-01-0755.

[17] C Landstrom, L Josefsson, TWalker, et al. Aerodynamic effects of different tire models on a sedan type passenger car. SAE Technical Papers, 2012-01-0169.

[18] A Guzman, Y C Cho, J Tripp, et al. Further analyses on prediction of automotive spinning wheel flowfield with full width moving belt wind tunnel results. SAE Technical Papers, 2017-01-1519.

[19] R Eleanor, W Duncan. Investigation of wheelhouse flow interaction and the influence of lateral wheel displacement. Energies, 2019, 12(17): 1-29.

[20] F L Wang, Z S Yin, S Yan, et al. Validation of aerodynamic simulation and wind tunnel test of the New Buick Excelle GT. SAE Technical Papers, 2017-01-1512.

[21] L Axon, K Garry, J Howell. An evaluation of cfd for modelling the flow around stationary and rotating isolated wheels. SAE Technical Papers, 1998-98-0032.

[22] S Diasinos, T J Barber, G Doig. The effects of simplifications on isolated wheel aerodynamics. Journal of Wind Engineering and Industrial Aerodynamics, 2015, 146: 90-101.

[23] J McManus, X Zhang. A computational study of the flow around an isolated wheel in contact with the ground. Journal of Fluids Engineering, Transactions of the ASME, 2006, 128(3): 520-530.

[24] F Wittmeier, N Widdecke, J Wiedemann, et al. Tyre development from an aerodynamic perspective. ATZ Worldwide, 2013, 115(2): 42-48.

[25] G M Uddin, S M Arafat, A H Kazim, et al. Artificial intelligence-based Monte-Carlo numerical simulation of aerodynamics of tire grooves using computational fluid dynamics. Artificial Intelligence for Engineering Design, Analysis and Manufacturing: AIEDAM, 2019, 33(3): 302-316.

[26] R Palin, V Johnston, S Johnson, et al. The aerodynamic development of the Tesla Model S-part 1: Overview. SAE Technical Papers, 2012-01-0177.

[27] THobeika, S Sebben. CFD investigation on wheel rotation modelling. Journal of Wind Engineering and Industrial Aerodynamics, 2018, 174: 241-251.

[28] H Berg, A Brandt. Investigation of aerodynamic wheel design. Chalmars University of Technology, Gothenburg, Sweden, 2018.

[29] T Hobeika, S Sebben, C Landstrom. Investigation of the influence of tyre geometry on the aerodynamics of passenger cars. SAE International Journal of Passenger Cars - Mechanical Systems, 2013, 6(1): 316-325.

[30] T Hobeika, L Löfdahl, S Sebben. Study of different tyre simulation methods and effects on passenger car aerodynamics. The International Vehicle Aerodynamics Conference, Holywell, UK. October 14-15, 2014, 187-195.

[31] H C Zhou, C Liang, J Yang, et al. Biomimetic methods for improving tire skid resistance. Journal of Mechanical Engineering, 2015, 51(8): 125130,136. (in Chinese)

[32] H C Zhou, G L Wang, Y M Ding, et al. Effect of friction model and tire maneuvering on tire-pavement contact stress. Advances in Materials Science and Engineering, 2015: 632647.

[33] T Li, H Hemida, JY Zhang, et al. Comparisons of shear stress transport and detached eddy simulations of the flow around trains. Journal of Fluids Engineering, 2018, 140(11): 111108-111112. 
[34] H Sun, R Xiao, F Wang, et al. Analysis of the pump-turbine S characteristics using the detached eddy simulation method. Chinese Journal of Mechanical Engineering, 2015, 28(1): 115-122.

[35] M S Gritskevich, A V Garbaruk, J Schütze, et al. Development of DDES and IDDES formulations for the $\mathrm{k}-\omega$ shear stress transport model. Flow, Turbulence and Combustion, 2012, 88(3): 431-449.

[36] R D Knowles. Monoposto racecar wheel aerodynamics: investigation of nearwake structure and support-sting interference. Cranfield University, Bedford, UK, 2005
[37] A J Saddington, R D Knowles, K Knowles. Laser Doppler anemometry measurements in the near-wake of an isolated Formula One wheel. Experiments in Fluids, 2007, 42(5): 671-681.

[38] T Hobeika. Investigation of tyre geometry influence on road vehicle aerodynamics. Chalmars University of Technology, Gothenburg, Sweden, 2012

[39] J C R Hunt, A A Wray, P Moin. Eddies, streams, and convergence zones in turbulent flows. Center for Turbulence Research, Proceedings of the Summer Program, 1988: 193-208.

\section{Submit your manuscript to a SpringerOpen ${ }^{\circ}$ journal and benefit from:}

- Convenient online submission

- Rigorous peer review

- Open access: articles freely available online

- High visibility within the field

- Retaining the copyright to your article

Submit your next manuscript at $\boldsymbol{\nabla}$ springeropen.com 